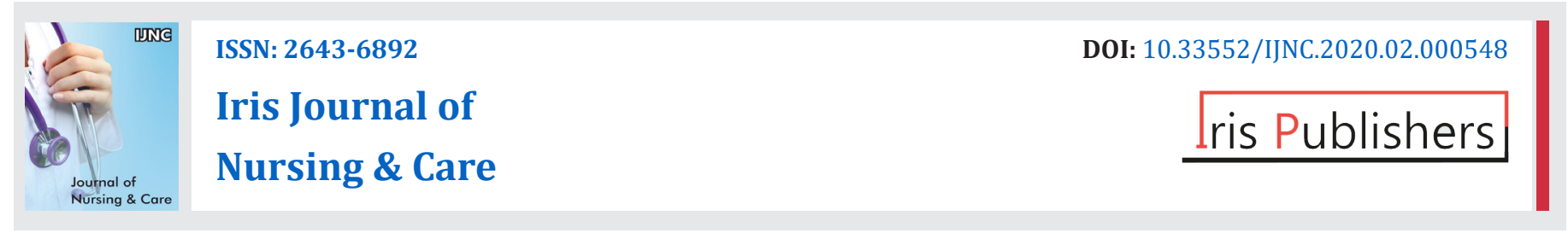

Mini Review

Copyright (C) All rights are reserved by Urmila Harold

\title{
Stop-Bang Tool for Sleep Apnea and Related Self-Efficacy
}

\author{
Urmila Harold* \\ Department of nursing, Graceland University, USA
}

*Corresponding author: Urmila Harold, Department of Nursing, Graceland University, USA.

Received Date: March 03, 2020

Published Date: March 18, 2020

\begin{abstract}
Obstructive sleep apnea (OSA) is a health issue that can cause many complications perioperatively if it is left undiagnosed or unidentified in the pre-operative assessment. The staff of the post-operative anesthesia care unit (PACU) at one of the hospitals in Missouri was managing the complications from the unidentified patients with OSA on an everyday basis. The problems associated with the complications included extended recovery room stay and an unexpected need to transfer the patients to a monitored bed. This DNP project aimed to assess the self-efficacy in the nurses after the education and implementation of the STOP-Bang tool to screen patients for OSA. The objectives of the project: To complete a pre-education self-efficacy survey utilizing the Evidence-Based Nursing Practice Self-Efficacy Survey, to educate and provide the resources about the STOP-Bang tool, and to evaluate the self-efficacy after the implementation of the STOP-Bang tool. The Iowa model of evidence-based practice was chosen for this project because the Iowa model guides step by step through the change process. An evidenced-based Self-Efficacy Survey tool was used to collect data. There was a significant increase in self-efficacy in nurses from pre-intervention to post-intervention. The data analysis demonstrated a significant increase in confidence scores from pre to post, $t(13)=-5.90, p<0.001$.
\end{abstract}

Keywords: Self efficacy; STOP-bang; Obstructive sleep apnea; Perioperative complications; Lowa model; Pre and post-anesthesia care unit

\section{Introduction}

Obstructive sleep apnea (OSA) causes many complications perioperatively if it is left unidentified in the pre-operative assessment. These complications include hypoxemia, myocardial injury, cardiac arrhythmias, and unexpected transfer to the intensive care unit [1]. STOP-Bang tool (Snore, Tired, Observed apnea, High Blood Pressure, Body Mass Index (BMI) more than 35, Age more than 50, Neck circumference more than $40 \mathrm{~cm}$, and male gender) was developed to screen the risk of sleep apnea in patients by Dr. Frances Chung of University Health Network [2].

\section{PICOT}

The PICOT question for this DNP scholarly project was: Among professional nurses in the pre and post-anesthesia care unit of a local hospital in Missouri, how does the education and implementation of the STOP-Bang tool increase evidence-based nursing practice self-efficacy over an eight-week time frame.

\section{Objectives and aims}

The DNP project aimed to assess the self-efficacy in the nurses after the education and implementation of the STOP-Bang tool in the pre-operative assessment. The author identified the following objectives to be serving as a guide to the DNP scholar during the project:

- $\quad$ To inform the stakeholders about the project and gain cooperation.

- $\quad$ To complete a pre-education self-efficacy survey utilizing the Evidence-Based Nursing Practice Self-Efficacy Scale (EBNPSE). 
- $\quad$ To educate and provide the resources about the STOPBang tool.

- To evaluate the self-efficacy after the implementation of the STOP-Bang tool.

\section{Theoretical framework}

The idea behind educating nurses about STOP-Bang was patient safety and care. Thus, an appropriate theoretical framework for the project, Watson's Caring theory, was selected which is based on the core concepts and ten Caritas. The core concepts of Jean Watson's Caring theory are relational caring for self and others, transpersonal caring, caring moment, multiple ways of knowing, reflective approach, caring is inclusive, caring changes self and others, and ten Caritas to put caring into action [3].

\section{Change model}

The Iowa model of evidence-based practice was chosen for this project because the Iowa model guides clearly step by step through the change process. The steps include the selection of a topic, forming a team, evidence retrieval, grading the evidence, developing an evidence-based practice standard, implementing EBP, and evaluation. Some of the nurses from the University of Iowa hospitals and clinics, along with the college of nursing, developed the Iowa model in the early 1990s to improve healthcare quality [4].

\section{Sample and setting}

The DNP project was implemented in a pre- and post-anesthesia care unit in one of the hospitals in Missouri. The hospital offered services to a variety of patients, including oncology, stroke, women's services, cardiac, trauma, and orthopedic surgery. The nurses working in these units were mostly BSN prepared nurses, male and female, and belonged to a diverse age group. These units had one charge nurse each. There were full time and part-time nurses that float back and forth in pre- and post-operative units. The culture of the units was based on cooperation and team building. Selection criteria included all the nurses responsible for patient assessment and none of the nurses excluded from the project.

\section{Intervention}

In the first week, the self-efficacy survey was administered after a brief introduction to staff who were willing to participate in the project. The author evaluated the self-efficacy before and after the implementation of the STOP-Bang by using the Evidence-Based Nursing Practice Self-Efficacy (EBNPSE) scale survey tool. The EBNPSE tool was developed by nurses from the hospitals of Iowa and clinics in 2009 to assess participants' confidence in completing the practice by self-rating $[5,6]$. The surveys were anonymous and confidential. The participants were asked to write a unique identifier instead of their names. In the second week, the author actualized the educational plan. Education on the STOP-Bang tool was provided three to four different times to ensure the complete coverage of nurses that work in the pre- and post-anesthesia care unit. From the third through the sixth week, the nurses assessed the patients using the STOP-Bang screening tool for high risk for OSA and post-operative complications. Resources and support were provided to the nurses if they had any questions. The collected data from the pre- and post-education EBNPSE survey was evaluated and analyzed by using SPSS version 25 (Armonk, NY: IBM Corp.).

\section{Findings}

Out of 17 surveys, there were only 14 valid surveys that could be used for data analysis. After the assumption of abnormality was met, repeated-measures t-test was done. Means (M) and standard deviations (SD) were reported for the t-test analysis. Statistical significance was assumed at an alpha value of 0.05 . All analyses were conducted using SPSS Version 25 (Armonk, NY: IBM Corp.). Repeated-measures t-test found that there was a statistically significant increase in total survey scores from pre-intervention (M $=42.38, \mathrm{SD}=27.51)$ to post-intervention $(\mathrm{M}=85.87, \mathrm{SD}=11.08)$.

\section{Conclusion}

The significant statistical increase in self-efficacy in the staff demonstrated the effectiveness of the education and implementation of the STOP-Bang tool.

\section{Acknowledgement}

I am thankful to my mentors and staff who helped and participated in this project.

\section{Conflict of Interest}

Author declare no conflict of interest.

\section{References}

1. Hai F, Porhomayon J, Vermont L, Frydrych L, Jaoude P, et al. (2014) Original contribution: Postoperative complications in patients with obstructive sleep apnea: a meta-analysis. Journal of Clinical Anesthesia 26(8): 591-600.

2. Toronto Western Hospital (2018) STOP Bang Questionnaire.

3. Watson Caring Science Institute (2018) Caring science theory.

4. Buckwalter KC, Cullen L, Hanrahan K, Kleiner C, Mc Carthy AM, et al. (2017) Iowa model of evidence-based practice: revisions and validation. Worldviews on Evidence-Based Nursing 14(3): 175-182.

5. Tucker SJ, Olson ME, Frusti DK (2009) Evidence-based practice selfefficacy scale: preliminary reliability and validity. Clinical Nurse Specialist 23(4): 207-215.

6. Iowa Model Collaborative, Kathleen C Buckwalter, Laura Cullen, Kirsten Hanrahan, Charmaine Kleiber, et al. (2017) Iowa model of evidencebased practice: Revisions and validation. Worldviews Evid Based Nurs 14(3): 175-182. 